\title{
A PAUCITY ESTIMATE RELATED TO NEWTON SUMS OF ODD DEGREE
}

\author{
JÖRG BRÜDERN AND OLIVIER ROBERT
}

Abstract. Paucity is established for a system of diagonal diophantine equations, in which the degrees are the odd numbers in ascending order.

§1. Introduction. Newton's power sums

$$
s_{h}=X_{0}^{h}+X_{1}^{h}+\cdots+X_{k}^{h}
$$

play a prominent role in the theory of symmetric functions. Diophantine equations involving such sums have also been studied extensively. The prime example is certainly the system of equations

$$
x_{0}^{h}+x_{1}^{h}+\cdots+x_{m}^{h}=y_{0}^{h}+y_{1}^{h}+\cdots+y_{m}^{h} \quad(1 \leqslant h \leqslant k)
$$

in which now $m$ is at least as large as $k$. Estimates for the number of its integral solutions inside a box are known as Vinogradov's mean value theorem, and are significant ingredients for the treatment of Waring's problem as well as for the determination of zero-free regions of Riemann's zeta function. Perhaps less commonly known, the allied problem where only odd degree equations occur is also of assistance in various fields of application. We refer to [7] for just one example. Thus, we now study the system of equations

$$
x_{0}^{2 j-1}+x_{1}^{2 j-1}+\cdots+x_{k}^{2 j-1}=y_{0}^{2 j-1}+y_{1}^{2 j-1}+\cdots+y_{k}^{2 j-1} \quad(1 \leqslant j \leqslant k) \text {. }
$$

A solution of (3) in which the $x_{0}, \ldots, x_{k}$ are a permutation of the $y_{0}, \ldots, y_{k}$ is referred to as trivial. We are interested in an upper bound for the number $U_{k}(B)$ of non-trivial integer solutions of (3) within the box

$$
1 \leqslant x_{i} \leqslant B, \quad 1 \leqslant y_{i} \leqslant B \quad(0 \leqslant i \leqslant k) .
$$

THEOREM. Let $k \geqslant 3$. Then there is a number $\lambda_{k}$ with $\lambda_{k}<k+1$ and $\lambda_{k}=$ $\frac{6}{7} k+O(1)$, and such that $U_{k}(B) \ll B^{\lambda_{k}}$. Moreover, one has $U_{3}(B) \ll B^{34 / 9+\varepsilon}$.

An explicit admissible choice for $\lambda_{k}$ is presented at the end of $\S 4$. To appreciate the strength of this estimate, notice that there are $(k+1) ! B^{k+1}+$ $O\left(B^{k}\right)$ trivial solutions of (3) in the range defined by (4). Although the methods 
in this paper would also apply to the case $k=2$, the conclusions are inferior to an asymptotic expansion for $U_{2}(B)$, in which the leading term is of size $B^{2}(\log B)^{5}$. Such a formula was predicted by conjectures of Manin and Peyre, and was confirmed in a celebrated memoir of la Bretèche [1]. The correct order of magnitude for $U_{2}(B)$ was determined earlier, by Vaughan and Wooley [8]. Their contribution has initiated much recent interest in paucity. The estimate announced in the theorem is the first of its kind for all $k \geqslant 3$. In these cases, one would not expect regularly distributed non-trivial solutions. However, when $k=3$, Choudhry [3] found a four-parameter family of non-trivial solutions of (3). Whether or not parametric families exist for larger $k$ remains an open question.

One may also take a projective point of view, counting rational points on the variety defined by the equations (3). It would then be more natural to count solutions in integers, rather than natural numbers. The system becomes more symmetric via the substitution $x_{k+1+j}=-y_{j}$, and now takes the shape

$$
\sum_{i=0}^{2 k+1} x_{i}^{2 j-1}=0 \quad(1 \leqslant j \leqslant k) .
$$

In this new context, a solution is non-trivial if $x_{i}+x_{j} \neq 0$ holds for all $0 \leqslant$ $i, j \leqslant 2 k+1$, and our method estimates the number of such solutions inside the box $\left|x_{i}\right| \leqslant B$ to the same precision as the theorem does for $U_{k}(B)$. We spare the reader the details.

It is perhaps of interest to compare our result with a similar one in the context of Vinogradov's mean value theorem. Vaughan and Wooley [9] considered the number $V_{k}(B)$ of non-trivial solutions of the system (2) with $m=k$, and established the estimate

$$
V_{k}(B) \ll B^{\sqrt{4 k+5}} .
$$

Note that Vaughan and Wooley discuss a system of $k$ equations in $2 k+2$ variables, as we do in our theorem. Their elementary argument resembles the idea of a torsor, and implicitly makes crucial use of the shift invariance of the system (2). The transition to certain auxiliary products that Vaughan and Wooley manufacture from identities that are in essence Newton's formulae is necessarily of a rather different nature when one is forced to work with power sums of odd degree only. It is mainly for this reason that our estimate is weaker than that of [9]. We derive a product identity suitable for our needs in the next section. The principal observation is a result on the algebraic dependence of the power sums of odd degree that seems to be due to Borchardt [2]. In §3, the proof of the theorem is prepared with a classification of the solutions to (3). In the final section, the estimation of $U_{k}(B)$ then proceeds along an avenue of attack that is perhaps the most novel feature of this note.

The notational conventions applied in this paper are standard, or otherwise introduced at the appropriate stage of the argument. Whenever the letter $\varepsilon$ occurs in a statement, it is asserted that $\varepsilon$ is a positive real number, and that the statement is valid for any positive value assigned to $\varepsilon$. Implicit constants suppressed by the use of Vinogradov's or Landau's well-known symbols may depend on $\varepsilon$ and $k$. 
§2. Preparatory algebra. In our first lemma we shall be concerned with polynomials in indeterminates $S_{1}, S_{3}, S_{5}, \ldots, S_{2 k-1}$. The weight of the monomial $S_{1}^{j_{1}} S_{3}^{j_{3}} \cdots S_{2 k-1}^{j_{2 k-1}}$ is the number $j_{1}+3 j_{3}+5 j_{5}+\cdots+(2 k-$ 1) $j_{2 k-1}$. The weight of a polynomial in these indeterminates is the largest weight of a monomial that occurs with a non-zero coefficient.

LEMMA 1. There exists a polynomial $P_{k} \in \mathbb{Q}\left[S_{1}, S_{3}, \ldots, S_{2 k-1}\right]$ of weight $\frac{1}{2} k(k+1)$ such that

$$
P_{k}\left(s_{1}\left(X_{1}, \ldots, X_{k-1}\right), s_{3}\left(X_{1}, \ldots, X_{k-1}\right), \ldots, s_{2 k-1}\left(X_{1}, \ldots, X_{k-1}\right)\right)=0 .
$$

This is Satz 1 of Perron [5], in slightly different notation, but a result of this type, apparently, was first obtained by Borchardt [2]. The interesting aspect of Perron's approach is that his method is constructive. Thus, one may compute $P_{k}$ for small values of $k$, beyond the obvious $P_{2}=S_{3}-S_{1}^{3}$. See Perron [6] for historical comments and also Foulkes [4] for related work, including formulae for $P_{3}$ and $P_{4}$.

We now introduce two further indeterminates, and for convenience write $\mathbf{X}=$ $\left(X_{0}, X_{1}, \ldots, X_{k}\right)$ and $\mathbf{X}^{\prime}=\left(X_{1}, X_{2}, \ldots, X_{k-1}\right)$. Consider the polynomial $Q \in \mathbb{Q}\left[X_{0}, \ldots, X_{k}\right]$ defined by

$$
Q(\mathbf{X})=P_{k}\left(s_{1}(\mathbf{X}), s_{3}(\mathbf{X}), \ldots, s_{2 k-1}(\mathbf{X})\right) .
$$

For odd $h \in \mathbb{N}$, one has $s_{h}(\mathbf{X}) \equiv s_{h}\left(\mathbf{X}^{\prime}\right) \bmod X_{0}+X_{k}$, whence $X_{0}+X_{k}$ divides $Q(\mathbf{X})$. By symmetry in the $X_{j}$, it follows that whenever $i \neq j$, then $X_{i}+X_{j}$ divides $Q(\mathbf{X})$, and so will then the product

$$
\Pi_{k}(\mathbf{X})=\prod_{0 \leqslant i<j \leqslant k}\left(X_{i}+X_{j}\right)
$$

This product is a homogenous polynomial of degree $\frac{1}{2} k(k+1)$, and, by Lemma $1, Q(\mathbf{X})$ has the same total degree. It follows that $\Pi_{k}$ is a rational multiple of $Q$. In Lemma 1 , however, $P_{k}$ is not unique, and one may replace $P_{k}$ by any non-zero rational multiple. Hence, we may suppose from now on that $P_{k}$ is chosen such that

$$
P_{k}\left(s_{1}(\mathbf{X}), s_{3}(\mathbf{X}), \ldots, s_{2 k-1}(\mathbf{X})\right)=\Pi_{k}(\mathbf{X}) .
$$

Lemma 2. Suppose that $\mathbf{x}, \mathbf{y} \in \mathbb{C}^{k+1}$ satisfy the equations (3). Then $\Pi_{k}(\mathbf{x})=\Pi_{k}(\mathbf{y})$.

Proof. By hypothesis, $s_{2 j-1}(\mathbf{x})=s_{2 j-1}(\mathbf{y})$ for $1 \leqslant j \leqslant k$. The lemma now follows from (6).

It is useful to observe that Lemma 2 yields further product relations with fewer factors. 
LEMMA 3. Suppose that $\mathbf{x}, \mathbf{y} \in \mathbb{C}^{k+1}$ satisfy the simultaneous equations (3). Moreover, suppose that $x_{i}+x_{j} \neq 0, y_{i}+y_{j} \neq 0$ for $0 \leqslant i<j \leqslant k$. Then

$$
\prod_{j=1}^{k}\left(x_{0}+x_{j}\right)\left(y_{j}-x_{0}\right)=\prod_{j=1}^{k}\left(y_{0}+y_{j}\right)\left(x_{j}-y_{0}\right) \text {. }
$$

Proof. By Lemma $2, \Pi_{k}(\mathbf{x})=\Pi_{k}(\mathbf{y})$, and by hypothesis, this number is nonzero. Another solution of (3) is obtained by substituting $-y_{0}$ for $x_{0}$, and $-x_{0}$ for $y_{0}$. By Lemma 2 again,

$$
\Pi_{k}\left(\left(-y_{0}, x_{1}, \ldots, x_{k}\right)\right)=\Pi_{k}\left(\left(-x_{0}, y_{1}, \ldots, y_{k}\right)\right) .
$$

It follows that

$$
\Pi_{k}\left(\left(-y_{0}, x_{1}, \ldots, x_{k}\right)\right) / \Pi_{k}(\mathbf{x})=\Pi_{k}\left(\left(-x_{0}, y_{1}, \ldots, y_{k}\right)\right) / \Pi_{k}(\mathbf{y}),
$$

and a rearrangement of terms yields (7).

There is another important application of (6) which will help identifying trivial solutions. It will be useful to formulate this for power sums in $k$ variables.

LEMMA 4. Let $\mathbf{x}=\left(x_{1}, \ldots, x_{k}\right)$ and $\mathbf{y}=\left(y_{1}, \ldots, y_{k}\right)$ be vectors of complex numbers, with $x_{i}+x_{j} \neq 0$ for all $1 \leqslant i<j \leqslant k$. Suppose that $s_{2 j-1}(\mathbf{x})=s_{2 j-1}(\mathbf{y})$ for $1 \leqslant j \leqslant k$. Then, the entries in $\mathbf{x}$ are a permutation of the entries in $\mathbf{y}$.

Proof. Let $t$ be a complex variable. We substitute $X_{0}=t$ and $X_{j}=x_{j}$ for $1 \leqslant j \leqslant k$ in (6). Then

$$
P_{k}\left(s_{1}(\mathbf{x})+t, s_{3}(\mathbf{x})+t^{3}, \ldots, s_{2 k-1}(\mathbf{x})+t^{2 k-1}\right)=p_{k}(t ; \mathbf{x}) \prod_{1 \leqslant i<j \leqslant k}\left(x_{i}+x_{j}\right)
$$

where

$$
p_{k}(t ; \mathbf{x})=\prod_{j=1}^{k}\left(t+x_{j}\right)=t^{k}+\sigma_{1}(\mathbf{x}) t^{k-1}+\cdots+\sigma_{k}(\mathbf{x}) t^{0}
$$

Here, the $\sigma_{j}$ denote the elementary symmetric functions. By hypothesis, replacing $\mathbf{x}$ with $\mathbf{y}$ will not change the left-hand side of (8). It follows that

$$
p_{k}(t ; \mathbf{x}) \prod_{1 \leqslant i<j \leqslant k}\left(x_{i}+x_{j}\right)=p_{k}(t ; \mathbf{y}) \prod_{1 \leqslant i<j \leqslant k}\left(y_{i}+y_{j}\right) .
$$

By hypothesis, the product of the $x_{i}+x_{j}$ on the left-hand side here does not vanish. Comparing coefficients at powers of $t$, it first follows that the product of the $y_{i}+y_{j}$ on the right is also non-zero, and then that $\sigma_{j}(\mathbf{x})=\sigma_{j}(\mathbf{y})$ holds for $1 \leqslant j \leqslant k$. It follows that $p_{k}(t ; \mathbf{x})=p_{k}(t, \mathbf{y})$. Considering the roots of these polynomials yields the desired conclusion. 
LEMMA 5. Let $\mathbf{x}, \mathbf{y} \in \mathbb{C}^{k+1}$, and suppose that the simultaneous equations (3) hold. Moreover, suppose that there are numbers $0 \leqslant i \leqslant k, 0 \leqslant j \leqslant k$ with $x_{i}=$ $y_{j}$. Then, this solution of (3) is trivial, or else there are $l, m$ with $0 \leqslant l, m \leqslant k$, $l \neq i \neq m$ with $x_{l}=-x_{m}$.

Proof. By symmetry, we may suppose that $x_{0}=y_{0}$. The conclusion of Lemma 5 now follows immediately from Lemma 4.

$\S 3$. Preparatory arithmetic. In this section, we investigate some of the diophantine consequences of the work in the previous section. Consider the diophantine system (3), and suppose that for exactly $k+1$ of the variables $x_{i}, y_{j}$ integer values have already been assigned in accordance with (4). Let $N$ denote the number of solutions to (3) in the remaining $k+1$ variables.

LEMMA 6. In the notation introduced above, one has $N \ll B^{\varepsilon}$.

Proof. Suppose that $l+1$ of the variables $x_{i}$, and $k-l$ of the variables $y_{j}$ have their fixed values assigned. By symmetry, we may suppose that at least one of the $x_{i}$ has a value assigned, whence $l \geqslant 0$. Again by symmetry, we may suppose that $x_{0}, x_{1}, \ldots, x_{l}, y_{l+1}, \ldots, y_{k}$ are the numbers that are fixed. If some of the $x_{i}$ in this list coincides with some $y_{j}$ in the list (if any), then by Lemma 5 all such solutions to (3) in positive integers are trivial. Hence, in that case, we have $N \ll 1$. In the contrary case where $x_{i} \neq y_{j}$ for $0 \leqslant i \leqslant l<j \leqslant k$, write $x_{i}^{\prime}=x_{i}$ for $0 \leqslant i \leqslant l$, and $x_{i}^{\prime}=-y_{i}$ for $l<i \leqslant k$. Similarly, let $y_{i}^{\prime}=y_{i}$ for $0 \leqslant i \leqslant l$ and $y_{i}^{\prime}=-x_{i}$ for $l<i \leqslant k$. Then (3) holds with $x_{i}^{\prime}, y_{i}^{\prime}$ for $x_{i}$, $y_{i}$, and one also has $x_{i}^{\prime}+x_{j}^{\prime} \neq 0$ for all pairs $i, j$. Hence, $\Pi_{k}\left(\mathbf{x}^{\prime}\right) \neq 0$. By Lemma 2, it follows that $N$ is bounded above by the number of $\mathbf{y}^{\prime} \in \mathbb{Z}^{k+1}$ with $\Pi_{k}\left(\mathbf{y}^{\prime}\right)=\Pi_{k}\left(\mathbf{x}^{\prime}\right)$. In particular, for any pair $i, j$ with $i<j$, the number $y_{i}^{\prime}+y_{j}^{\prime}$ is non-zero and a divisor of the non-zero number $\Pi_{k}\left(\mathbf{x}^{\prime}\right)$. Moreover, recall that $\left|x_{i}^{\prime}\right| \leqslant B$, whence $\left|\Pi_{k}\left(\mathbf{x}^{\prime}\right)\right| \leqslant(2 B)^{(1 / 2) k(k+1)}$. A divisor function estimate now shows that there are no more than $O\left(B^{\varepsilon}\right)$ possibilities for each of the numbers $y_{i}^{\prime}+y_{j}^{\prime}$. Since these will determine the $y_{j}^{\prime}$, we deduce that $N \ll B^{\varepsilon}$, as required.

Now suppose that $\mathbf{x}, \mathbf{y}$ is a non-trivial solution of (3) in positive integers. Our next goal is a parametrization of these solutions. By Lemma 5, we have $x_{0} \neq y_{j}$ and $y_{0} \neq x_{j}$ for $1 \leqslant j \leqslant k$. By Lemma 3 , the identity (7) holds, and the products there are non-zero. It will be convenient to introduce the non-zero integers

$$
\begin{gathered}
w_{j}=x_{0}+x_{j}, \quad v_{j}=y_{0}+y_{j}, \quad u_{j}=y_{j}-x_{0}, \\
t_{j}=x_{j}-y_{0} \quad(1 \leqslant j \leqslant k) .
\end{gathered}
$$

The equation (7) then reads

$$
w_{1} w_{2} \cdots w_{k} u_{1} u_{2} \cdots u_{k}=v_{1} v_{2} \cdots v_{k} t_{1} t_{2} \cdots t_{k}
$$

We describe a recursive process to cancel factors in these products. For $\mathbf{i}, \mathbf{j} \in \mathbb{N}^{2}$ we write $\mathbf{i}<\mathbf{j}$ if either $i_{1}<j_{1}$, or $i_{1}=j_{1}$ and $i_{2}<j_{2}$. 
Now put $a_{11}=\left(w_{1} ; v_{1}\right), b_{11}=\left(u_{1} ; t_{1}\right)$, and then for $1 \leqslant i_{1}, i_{2} \leqslant k$ define inductively

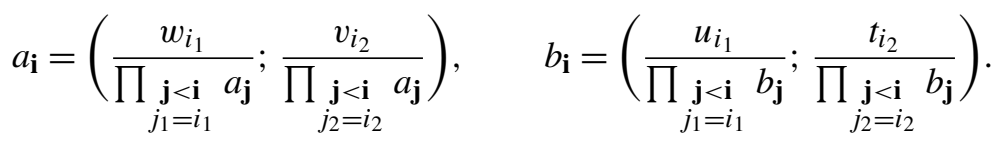

Now put

$$
v_{j}^{\prime \prime}=\prod_{i=1}^{k} a_{i j}, \quad t_{j}^{\prime \prime}=\prod_{i=1}^{k} b_{i j}, \quad w_{i}^{\prime \prime}=\prod_{j=1}^{k} a_{i j}, \quad u_{i}^{\prime \prime}=\prod_{j=1}^{k} b_{i j},
$$

and then write

$$
v_{j}=v_{j}^{\prime} v_{j}^{\prime \prime}, \quad u_{j}=u_{j}^{\prime} u_{j}^{\prime \prime}, \quad w_{i}=w_{i}^{\prime} w_{i}^{\prime \prime}, \quad t_{i}=t_{i}^{\prime} t_{i}^{\prime \prime} .
$$

Any common factors between a $w_{l}$ and a $v_{h}$ in (10) have now been coded into the numbers $a_{\mathbf{i}}$, and likewise for $u_{l}$ and $t_{h}$ which have their common factors coded into the $b_{\mathbf{i}}$. We now repeat this argument, and consider remaining common factors between $u_{l}$ and $v_{h}$, and between $w_{l}$ and $t_{h}$. Thus, we now put $c_{11}=\left(u_{1}^{\prime} ; v_{1}^{\prime}\right), d_{11}=\left(w_{1}^{\prime}, t_{1}^{\prime}\right)$, and then for $1 \leqslant i_{1}, i_{2} \leqslant k$ define inductively

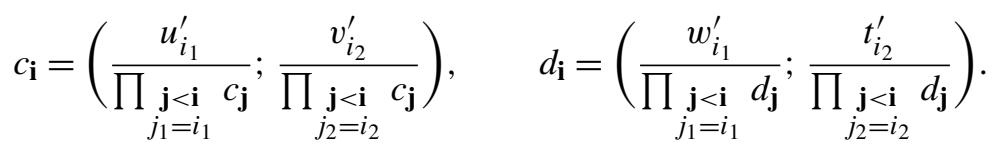

All prime factors dividing the left-hand side of (10) have now been assigned to a factor $a_{\mathbf{i}}, b_{\mathbf{i}}, c_{\mathbf{i}}$ or $d_{\mathbf{i}}$, and by unique factorization, it follows that

$$
\begin{array}{rlrl}
v_{j} & =\prod_{i=1}^{k} a_{i j} c_{i j}, & \left|t_{j}\right|=\prod_{i=1}^{k} d_{i j} b_{i j}, \\
w_{i}=\prod_{j=1}^{k} a_{i j} d_{i j}, & \left|u_{i}\right|=\prod_{j=1}^{k} c_{i j} b_{i j}
\end{array}
$$

holds for $1 \leqslant i, j \leqslant k$. We may summarize these deliberations as follows: with any non-trivial solution of (3) in positive integers, there are four $k \times k$ matrices $A=\left(a_{i j}\right), B=\left(b_{i j}\right), C=\left(c_{i j}\right)$ and $D=\left(d_{i j}\right)$ with positive integral entries, and these matrices determine the numbers $v_{j}, w_{j},\left|u_{j}\right|,\left|t_{j}\right|$. Another view of this is a $2 k \times 2 k$ matrix-like diagram

\begin{tabular}{c|cccccc} 
& $v_{1}$ & $\ldots$ & $v_{k}$ & $\left|t_{1}\right|$ & $\ldots$ & $\left|t_{k}\right|$ \\
\hline$w_{1}$ & $a_{11}$ & $\ldots$ & $a_{1 k}$ & $d_{11}$ & $\ldots$ & $d_{1 k}$ \\
$\vdots$ & $\vdots$ & & $\vdots$ & $\vdots$ & & $\vdots$ \\
$w_{k}$ & $a_{k 1}$ & $\ldots$ & $a_{k k}$ & $d_{k 1}$ & $\ldots$ & $d_{k k}$ \\
$\left|u_{1}\right|$ & $c_{11}$ & $\ldots$ & $c_{1 k}$ & $b_{11}$ & $\ldots$ & $b_{1 k}$ \\
$\vdots$ & $\vdots$ & & $\vdots$ & $\vdots$ & & $\vdots$ \\
$\left|u_{k}\right|$ & $c_{k 1}$ & $\ldots$ & $c_{k k}$ & $b_{k 1}$ & $\ldots$ & $b_{k k}$
\end{tabular}

where the product of the entries in a row gives the number on the left, and the product of the entries in a column is the number on top. 
$\S 4$. Counting non-trivial solutions. We now embark on the main counting argument. Let $k \geqslant 3$, and define numbers $J=[k / 2]$ and $I=k-1-J$. Then $I=[k / 2]$ when $k$ is odd, and $I=\frac{1}{2} k-1$ when $k$ is even. From now on, let $\mathscr{I}$, $\mathscr{J}$ denote subsets of $\{1, \ldots, k\}$ with $\# \mathscr{I}=I, \# \mathscr{J}=J$. For any non-trivial solution of (3) in natural numbers, we consider

$$
z_{1}(\mathscr{I}, \mathscr{J})=\prod_{i \in \mathscr{I}, j \in \mathscr{J}} a_{i j} \quad \text { and } \quad z_{1}=\max _{\mathscr{I}, \mathscr{J}} z_{1}(\mathscr{I}, \mathscr{J}) .
$$

We begin by counting non-trivial solutions of (3) and (4) for which $z_{1}$ takes a prescribed value. First choose $\mathscr{I}, \mathscr{J}$ with $z_{1}=z_{1}(\mathscr{I}, \mathscr{J})$. By (11), the numbers

$$
\prod_{i \in \mathscr{I}} a_{i j}, \quad \prod_{j \in \mathscr{J}} a_{i j}
$$

are divisors of $v_{j}$ and $w_{i}$, respectively. Moreover, by (9) and (4), we have $v_{j} \leqslant 2 B, w_{i} \leqslant 2 B$. On the one hand, this shows that $z_{1}(\mathscr{I}, \mathscr{J})$ divides the product of the $v_{j}$ with $j \in \mathscr{J}$, and hence that $z_{1} \leqslant(2 B)^{J}$. Therefore, there are no more than $O\left(B^{\varepsilon}\right)$ choices for $a_{i j}$ with $i \in \mathscr{I}, j \in \mathscr{J}$, as one readily confirms by invoking a divisor function estimate. On the other hand, this also implies that

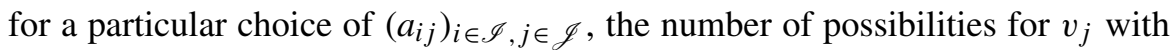
$j \in \mathscr{J}$ is $O\left(B^{J} z_{1}^{-1}\right)$. Similarly, the number of choices for $w_{i}$ with $i \in \mathscr{I}$ is $O\left(B^{I} z_{1}^{-1}\right)$. Now fix one of the $O\left(B^{2}\right)$ choices for $x_{0}, y_{0}$. Then, by (9), this fixes also $x_{i}, y_{j}$ with $i \in \mathscr{I}, j \in \mathscr{J}$, so that $2+(I+J)=k+1$ of the variables in (3) are determined. Lemma 6 shows that this leaves $O\left(B^{\varepsilon}\right)$ possibilities for the remaining variables. On collecting together, we see that at most $O\left(B^{k+1+\varepsilon} z_{1}^{-2}\right)$ non-trivial solutions of (3) and (4) have $z_{1}(\mathscr{I}, \mathscr{J})=z_{1}$.

Fix a parameter $Z$ with $1 \leqslant Z \leqslant B^{k / 2}$. A non-trivial solution of (3) with $z_{1}>Z$ and counted by $U(B)$ is said to be of type I, and we write $U^{(\mathrm{I})}(B)$ for their number. Then, the above estimate implies that

$$
U^{(\mathrm{I})}(B) \ll \sum_{z \geqslant Z} B^{k+1+\varepsilon} z^{-2} \ll B^{k+1+\varepsilon} Z^{-1} .
$$

We perform a similar analysis with the matrix $C$. In this case, we choose $\mathscr{I}$, as above, but with the additional constraint that these two sets are disjoint. Thus, we now put

$$
z_{2}(\mathscr{I}, \mathscr{J})=\prod_{i \in \mathscr{I}, j \in \mathscr{J}} c_{i j} \quad \text { and } \quad z_{2}=\max _{\mathscr{I} \cap \mathscr{J}=\emptyset} z_{2}(\mathscr{I}, \mathscr{J}) \text {. }
$$

We say that a non-trivial solution of (3) within the box (4) with $z_{2}>Z$ is of type II, and we write $U^{(\mathrm{II})}(B)$ for the number of such solutions. An estimate for this number is readily obtained by imitating the preceding argument. Consider the solutions with $z_{2}(\mathscr{I}, \mathscr{J})=z_{2}$, say. Then, as above, this leaves $O\left(B^{I+J+\varepsilon} z_{2}^{-2}\right)$ for $u_{i}, v_{j}$ with $i \in \mathscr{I}, j \in \mathscr{J}$. Now fix one of the $O\left(B^{2}\right)$ choices for $x_{0}, y_{0}$. Then, by (9), we have fixed $x_{0}, y_{0}$ and $k-1$ of the variables $y_{1}, \ldots, y_{k}$. Note that this makes crucial use of the fact that $\mathscr{I}$ and $\mathscr{J}$ are 
disjoint. By Lemma 6, there are no more than $O\left(B^{\varepsilon}\right)$ choices for the remaining variables. Hence, we again find that at most $O\left(B^{k+1+\varepsilon} z_{2}^{-2}\right)$ non-trivial solutions of (3) and (4) have $z_{2}(\mathscr{I}, \mathscr{J})=z_{2}$, and as before we deduce the final estimate

$$
U^{(\mathrm{II})}(B) \ll B^{k+1+\varepsilon} Z^{-1} .
$$

It remains to count non-trivial solutions of (3) with (4) that are neither of type I nor II. In preparation for this task, we show that for such solutions one has the inequalities

$$
\prod_{1 \leqslant i, j \leqslant k} a_{i j} \ll Z^{\beta}, \quad \prod_{\substack{1 \leqslant i, j \leqslant k \\ i \neq j}} c_{i j} \ll Z^{\gamma}
$$

with $\beta=k^{2} /(I J)$ and

$$
\gamma=\frac{4 k}{k-1} \quad(k \text { odd }), \quad \gamma=\frac{4(k-1)}{k-2} \quad(k \text { even }) .
$$

To demonstrate the first inequality in (13), first note that there are $\left(\begin{array}{l}k \\ I\end{array}\right)$ possibilities to position $\mathscr{I}$ in $\{1, \ldots, k\}$, and $\left(\begin{array}{c}k \\ J\end{array}\right)$ choices for $\mathscr{J}$. Since we are not considering solutions of type I, we have $z_{1}(\mathscr{I}, \mathscr{J})<Z$ for each of these choices. This shows that

$$
\prod_{\mathscr{I}, \mathscr{J}} z_{1}(\mathscr{I}, \mathscr{J}) \leqslant Z^{\left({ }_{I}^{k}\right)\left({ }_{J}^{k}\right)}
$$

where the product extends over all admissible choices for $\mathscr{I}, \mathscr{J}$.

Now consider a particular pair $(i, j)$ with $1 \leqslant i, j \leqslant k$. The number of choices for $\mathscr{I}, \mathscr{J}$ with $(i, j) \in \mathscr{I} \times \mathscr{J}$ is $\left(\begin{array}{c}k-1 \\ I-1\end{array}\right)\left(\begin{array}{c}k-1 \\ J-1\end{array}\right)$ because there are $\left(\begin{array}{c}k-1 \\ I-1\end{array}\right)$ possibilities for $\mathscr{I}$ with $i \in \mathscr{I}$, and similarly for $\mathscr{J}$. This implies that

$$
\prod_{\mathscr{I}, \mathscr{J}} z_{1}(\mathscr{I}, \mathscr{J})=\prod_{1 \leqslant i, j \leqslant k} a_{i j}^{\left(\begin{array}{l}
k-1 \\
I-1
\end{array}\right)\left(\begin{array}{c}
k-1 \\
J-1
\end{array}\right)},
$$

and by comparison with the previous estimate we deduce that the first inequality in (13) is valid with

$$
\beta=\frac{\left(\begin{array}{l}
k \\
I
\end{array}\right)\left(\begin{array}{l}
k \\
J
\end{array}\right)}{\left(\begin{array}{l}
k-1 \\
I-1
\end{array}\right)\left(\begin{array}{l}
k-1 \\
J-1
\end{array}\right)}=\frac{k^{2}}{I J},
$$

as required.

A similar argument yields the second inequality in (13). For non-trivial solutions of (3) and (4) that are not of type II, one has $z_{2}(\mathscr{I}, \mathscr{J}) \leqslant Z$ for all disjoint admissible pairs $\mathscr{I}, \mathscr{J}$. To compute the number of possible choices for $\mathscr{I}, \mathscr{J}$, we may first fix one of the $\left(\begin{array}{c}k \\ I\end{array}\right)$ choices for $\mathscr{I}$. This leaves $k-I=J+1$ numbers in $\{1, \ldots, k\}$ that are not in $\mathscr{I}$, and $\mathscr{J}$ must be a subset of this set, with $J$ elements. This leaves $k-I$ possible choices for $\mathscr{J}$. It follows that

$$
\prod_{\mathscr{I} \cap \mathscr{J}=\emptyset} z_{2}(\mathscr{I}, \mathscr{J}) \leqslant Z^{\left({ }_{I}^{k}\right)(k-I)} .
$$


However, we also have

$$
\prod_{\mathscr{I} \cap \mathscr{J}=\emptyset} z_{2}(\mathscr{I}, \mathscr{J})=\prod_{\substack{1 \leqslant i, j \leqslant k \\ i \neq j}} c_{i j}^{H}
$$

where $H=H(i, j)$ denotes the number of disjoint sets $\mathscr{I}, \mathscr{J}$ with $(i, j) \in$ $\mathscr{I} \times \mathscr{J}$. By symmetry, it is apparent that $H$ does not depend on the particular pair $i, j$. To position $\mathscr{I}$ in $\{1, \ldots, k\}$, we have to make $I-1$ choices different from $i$ and $j$, and since $i \neq j$, there are $\left(\begin{array}{c}k-2 \\ I-1\end{array}\right)$ choices for $\mathscr{I}$. There are then $k-I-1=J$ elements left in $\{1, \ldots, k\}$ that are different form $j$, and this leaves $J$ choices for $\mathscr{J}$. Hence, $H=J\left(\begin{array}{c}k-2 \\ I-1\end{array}\right)$. It now follows that the product in the second estimate of (13) is bounded by $Z^{\gamma}$ where $\gamma=\left(\begin{array}{c}k \\ I\end{array}\right)(k-I) H^{-1}$, and it is readily confirmed that this value of $\gamma$ coincides with the one given in (14).

We are ready to complete the counting procedure. Let $K$ denote a sufficiently large real number. Then, by (13), for any solution counted by $U_{k}(B)$ that is not of type I or II, at least one of the inequalities

$$
\prod_{1 \leqslant i<j \leqslant k} c_{i j} \leqslant K Z^{\gamma / 2}, \quad \prod_{1 \leqslant j<i \leqslant k} c_{i j} \leqslant K Z^{\gamma / 2}
$$

must hold. We say that a solution is of type III if it is not of type I or II, and the first inequality in (15) holds. The remaining solutions are said to be of type IV. Note that for type IV solutions, the second inequality in (15) holds. We let $U^{(\mathrm{III})}(B)$ and $U^{(\mathrm{IV})}(B)$ be the number of solutions of type III, type IV respectively.

We begin with the estimation of $U^{(\mathrm{III})}(B)$, and first fix one of the $O\left(B^{2}\right)$ choices for $x_{0}, y_{0}$. Then, for any solution of (3), we have $c_{i i} \mid v_{i}-u_{i}=x_{0}+y_{0}$, for $1 \leqslant i \leqslant k$. This leaves $O\left(B^{\varepsilon}\right)$ choices for $c_{11}, c_{22}, \ldots, c_{k k}$, of which we now fix one. By (13) and a divisor function estimate, there are $O\left(Z^{\beta+\varepsilon}\right)$ possible choices for the $a_{i j}$ with $1 \leqslant i, j \leqslant k$, and $O\left(Z^{\gamma / 2+\varepsilon}\right)$ choices for the $c_{i j}$ with $1 \leqslant i<j \leqslant k$, and we fix one of these choices now. Note that the upper triangular part, including the diagonal, of the matrix $C$ is now known, in particular its last column. By (11), this determines $v_{k}$, and therefore $y_{k}$. Then, however, $u_{k}$ is also known. Hence, there are no more than $O\left(B^{\varepsilon}\right)$ choices for the divisors $c_{k j}$ of $u_{k}$, for $1 \leqslant j \leqslant k-1$. We fix one of these choices. This completes the last row, and hence the penultimate column of $C$ so that $v_{k-1}$ is determined. This determines $y_{k-1}$ and $u_{k-1}$. As before, the entries of the $(k-1)$ th row are divisors of $u_{k-1}$, leaving $O\left(B^{\varepsilon}\right)$ choices for the missing entries. Repeating this argument $k-1$ times, one finds that there are no more than $O\left(B^{\varepsilon}\right)$ choices for $y_{1}, \ldots, y_{k}$. We have now fixed the $k+2$ variables $x_{0}$ and $y_{j}$ with $0 \leqslant j \leqslant k$, leaving $O\left(B^{\varepsilon}\right)$ choices for the remaining variables by Lemma 6. On collecting together, we infer that

$$
U^{(\mathrm{III})}(B) \ll B^{2+\varepsilon} Z^{\beta+\gamma / 2} .
$$

For solutions of type IV, the previous argument also applies save that one seeds the lower triangular part of $C$ into the beginning of the argument. Then $v_{1}$ 
will be determined first, and one constructs the upper triangular part on the fly, while fixing $y_{2}, y_{3}, \ldots$ recursively. The resulting estimate will be the same as for solutions of type III.

Recalling the bounds for the number of solutions of type I and II, we conclude that

$$
U_{k}(B) \ll B^{k+1+\varepsilon} Z^{-1}+B^{2+\varepsilon} Z^{\beta+\gamma / 2} .
$$

We balance terms with $Z=B^{\theta}$ where

$$
\theta=(k-1) /(1+\beta+\gamma / 2) .
$$

It is immediate that $\theta>0$, and that $\theta=\frac{1}{7} k+O(1)$. The first part of the theorem follows with $\lambda_{k}=k+1-\theta$.

\$5. A refinement. Some of the arguments applied in the previous section are rather crude, and call for an improvement. In particular, the variables $x_{0}$ and $y_{0}$ are chosen at random, and in the treatment of type III and type IV solutions we determined $k+2$ variables before using Lemma 6, although this would apply already when $k+1$ variables are fixed. However, attending to such matters will reduce the size of $\lambda_{k}$ in the theorem only by $O(1)$, which is not of great significance when $k$ is large. For small values of $k$, however, one may obtain rather better bounds, and we illustrate this with the case $k=3$ in this section.

The notation from the previous sections will be kept. We choose $i, j$ with $1 \leqslant i, j \leqslant 3$, and consider solutions of (3) and (4) for which $a_{i j}$ and $c_{j j}$ take a fixed value. In this situation, we first fix one of the $O(B)$ choices for $y_{0}$, and then observe that there are no more than $B /\left(a_{i j} c_{j j}\right)$ choices for $v_{j}$. This determines $y_{j}$. Now $c_{j j} \mid x_{0}-y_{j}$, leaving $O\left(B / c_{j j}\right)$ choices for $x_{0}$. Finally, $a_{i j} \mid x_{0}+x_{i}$, leaving $O\left(B / a_{i j}\right)$ choices for $x_{i}$. In total, this allows no more than $B^{4}\left(a_{i j} c_{j j}\right)^{-2}$ choices for the variables $x_{0}, x_{i}, y_{0}, y_{j}$, and Lemma 6 fixes the remaining ones, within $O\left(B^{\varepsilon}\right)$. A divisor sum argument then shows that the contribution to $U_{3}(B)$ from solutions with $a_{i j} c_{j j} \geqslant Z$ is $O\left(B^{4+\varepsilon} / Z\right)$. A very similar argument estimates the contribution from solutions with $c_{i j} c_{j j} \geqslant Z$ for some $i \neq j$ to the same precision. Hence, we have

$$
U_{3}(B) \ll B^{4+\varepsilon} Z^{-1}+U^{\prime}(B),
$$

where $U^{\prime}(B)$ is the number of solutions counted by $U_{3}(B)$ that satisfy the inequalities

$$
a_{i j} c_{j j}<Z \text { for } 1 \leqslant i, j \leqslant 3, \quad c_{i j} c_{j j}<Z \text { for } 1 \leqslant i, j \leqslant 3, i \neq j .
$$

Now consider solutions counted by $U^{\prime}(B)$ for which the numbers $c_{11}, c_{12}, c_{21}, c_{22}$ take a fixed value. We then choose all $a_{i 1}, a_{i 2}$ and $c_{31}, c_{32}$. There are $O\left(Z^{8}\left(c_{11} c_{22}\right)^{-4}\right)$ such choices, and the numbers $v_{1}, v_{2}$ are now determined. We pick one of the $O(B)$ values for $y_{0}$, and then compute $y_{j}=v_{j}-y_{0}$ for $j=1$ and 2 . Now, however, $c_{11} c_{12} \mid x_{0}-y_{1}$ and $c_{21} c_{22} \mid$ $x_{0}-y_{2}$, leaving $O\left(B\left(c_{11} c_{12} ; c_{21} c_{22}\right)\left(c_{11} c_{12} c_{21} c_{22}\right)^{-1}\right)$ choices for $x_{0}$. Now 
four variables in (3) are determined, and there are $O\left(B^{\varepsilon}\right)$ choices for the remaining ones. This yields

$$
U^{\prime}(B) \ll B^{2+\varepsilon} Z^{8} \sum\left(c_{11} c_{22}\right)^{-5}\left(c_{12} c_{21}\right)^{-1}\left(c_{11} c_{12} ; c_{21} c_{22}\right),
$$

where the sum is over all $c_{11}, c_{12}, c_{21}, c_{22}$ satisfying (16). A routine estimation then shows that $U^{\prime}(B) \ll B^{2+\varepsilon} Z^{8}$, and with $Z=B^{2 / 9}$ the bound $U_{3}(B) \ll$ $B^{34 / 9+\varepsilon}$ follows.

\section{References}

1. R. de la Bretèche, Répartition des points rationnels sur la cubique de Segre. Proc. Lond. Math. Soc. (3) 95 (2007), 69-155.

2. C. W. Borchardt, Über eine Eigenschaft der Potenzsummen ungerader Ordnung. In Monatsberichte der Berliner Akademie, (1857), 24-34.

3. A. Choudhry, The Diophantine system $\sum_{1}^{4} x_{i}^{r}=\sum_{1}^{4} y_{i}^{r}, r=1,3,5$. Bull. Calcutta Math. Soc. 83 (1991), 85-86.

4. H. O. Foulkes, Theorems of Kakeya and Pólya on power-sums. Math. Z. 65 (1956), 345-352.

5. O. Perron, Über die Abhängigkeit von Potenzsummen und einen Satz von Pólya. Math. Z. 63 (1955), 19-30.

6. O. Perron, Über Potenzsummen. Math. Z. 64 (1956), 103-114.

7. O. Robert, An analogue of van der Corput's $A^{5}$-process for exponential sums. Mathematika 49 (2002), $167-183$.

8. R. C. Vaughan and T. D. Wooley, A certain nonary cubic form and related equations. Duke Math. J. 80(3) (1995), 669-735.

9. R. C. Vaughan and T. D. Wooley, A special case of Vinogradov's mean value theorem. Acta Arith. 79 (1997), 193-204.

Jörg Brüdern,

Mathematisches Institut,

Bunsenstrasse 3-5,

D 37073 Göttingen,

Germany

E-mail: bruedern@uni-math.gwdg.de
Olivier Robert,

Institut Camille Jordan CNRS UMR 5208,

Université de Lyon

and

Université de Saint-Etienne,

23, rue du Dr P. Michelon, F-42000,

Saint-Etienne,

France

E-mail: olivier.robert@univ-st-etienne.fr 